[4] Z. R. Yang and R. Hamer, "Bio-basis function neural networks in protein data mining," Curr. Pharm. Res., vol. 13, pp. 1403-1413, 2007.

[5] C. Bishop, Neural Networks for Pattern Recognition. New York: Oxford Univ. Press, 1995

[6] R. O. Duda and P. E. Hart, Pattern Classification and Scene Analysis. New York: Wiley, 2002.

[7] M. Li and I. K. Sethi, "Confidence-based classifier design," Pattern Recognit., vol. 39, pp. 1230-1240, 2006.

[8] A. Madevska-Bogdanova, D. Nikolik, and L. Curfs, "Probabilistic SVM outputs for pattern recognition using analytical geometry," Neurocomputing, vol. 62, pp. 293-303, Dec. 2004.

[9] J. Platt, "Probabilistic outputs for SVMs and comparisons to regularized likelihood methods," in Advances in Large Margin Classifiers, P. J. Bartlett, B. Schölkopf, D. Schuurmans, and A. J. Smola, Eds. Cambridge, MA: MIT Press, 1999.

[10] B. Zadrozny and C. Elkan, "Transforming classifier scores into accurate multiclass probability estimates," in Proc 8th Int. Conf. Knowl. Discov. Data Mining (KDD'02), Edmonton, AB, Canada, Jul. 23-26, 2002, pp. 694-699.

[11] R. Francki, C. Fauquet, D. Knudson, and F. Brown, "Classification and nomenclature of viruses," Arch. Virol., vol. 2, p. 223, 1991.

[12] C. Steinkühler, A. Urbani, L. Tomei, G. Bisdiol, M. Sardana, E. Bianchi, A. Pessi, and R. De Francesco, "Activity of purified hepatitis $C$ virus protease NS3 on peptide substrates," J. Virol., vol. 70, pp. 6694-6700, 1996.

[13] A. Kwong, J. Kim, G. Rao, D. Lipovsek, and S. Raybuck, "Hepatitis C virus NS3/4A protease," Antiviral Res., vol. 40, pp. 1-18, 1998.

[14] T. Chambers, R. Weir, A. Grakoui, D. McCourt, F. Bazan, R. Fletterick, and C. Rice, "Evidence that the N-Terminal domain of nonstructural protein NS3 from Yellow Fever Virus is a serine proteiase responsible for site-specific cleavages in the viral polyprotein," Proc. Nat. Acad. Sci. USA, vol. 87, pp. 8898-8902, 1990.

[15] I. Schechter and A. Berger, "On the size of active sites in proteases. I. Papain.," Biochem. Bio. Res. Commun., vol. 27, pp. 157-162, 1967.

[16] J. Bukh, R. H. Purcell, and R. H. Miller, "At least 12 genotypes of hepatitis $\mathrm{C}$ virus predicted by sequence analysis of the putative $\mathrm{E} 1$ gene of isolates collected worldwide," Proc. Nat. Acad. Sci. USA, vol. 91, pp. 8234-8238, 1993

[17] E. Samokhvalov, M. Hijikata, R. Gylka, D. K. Lvov, and S. Mishiro, "Full-genome nucleotide sequence of a hepatitis $\mathrm{C}$ virus variant (isolate name VAT96) representing a new subtype within the genotype 2 (arbitrarily 2k)," Virus Genes, vol. 20, pp. 183-187, 2000

[18] L. Stuyver, W. Van Arnhem, A. Wyseur, F. Hernandez, E. Delaporte, and G. Maertens, "Classification of hepatitis $\mathrm{C}$ viruses based on phylogenetic analysis of the envelope 1 and nonstructural 5B regions and identification of five additional subtypes," Proc. Nat. Acad. Sci. USA, vol. 91, pp. 1013410138,1994

[19] A. Narayanan, X. Wu, and Z. R. Yang, "Mining viral protease data to extract cleavage knowledge," Bioinformat., vol. 18, pp. 5-13, 2002.

[20] C. Nello and J. Shaw-Taylor, An Introduction to Support Vector Machines, Cambridge, U.K.: Cambridge Univ. Press, 2000.

[21] R. Thomson, T. Hodgman, Z. R. Yang, and A. Doyle, "Characterising proteolytic cleavage site activity using bio-basis function neural networks," Bioinformatics, vol. 19, pp. 1741-1747, 2003.

[22] Z. R. Yang and R. Thomson, "Bio-basis function neural network for prediction of protease cleavage sites in proteins," IEEE Trans. Neural Netw., vol. 16, no. 1, pp. 263-274, Jan. 2005.

[23] M. O. Dayhoff, R. M. Schwartz, and B. C. Orcutt, "A model of evolutionary change in proteins. matrices for detecting distant relationships," Atlas Protein Seq. Str., vol. 5, pp. 345-358, 1978.

[24] D. J. MaCkay, "A practical Bayesian framework for backpropagation networks," Neural Comput., vol. 4, pp. 448-472, 1992.

[25] T. Joachims, "Making large-scale SVM learning practical," in Advances in Kernel Methods-Support Vector Learning, B. Scholkopf and C. Burges, Eds. Cambridge, MA: MIT Press, 1999.

[26] B. W. Matthews, "Comparison of the predicted and observed secondary structure of T4 phage lysozyme," Biochim. Biophys. Acta, vol. 405, pp. 442-451, 1975.

[27] C. E. Metz, "Basic principles of ROC analysis," Semin. Nucl.Med., vol. 8, pp. 283-298, 1978.

\section{Visualization of Back Pain Data-A 3-D Solution}

\author{
Gheorghita Ghinea, Simon Kent, Andrew O. Frank, \\ and Gurudain Singh Chana
}

\begin{abstract}
Traditional approaches to gathering and visualizing pain data rely on two-dimensional (2-D) human body models, where different types of sensation are recorded with various monochrome symbols. We propose an alternative that uses a three-dimensional (3-D) representation of the human body, which can be marked in color to visualize and record pain data.
\end{abstract}

Index Terms-Pain drawings, three-dimensional (3-D), visualization.

\section{INTRODUCTION}

Back pain is ubiquitous and seemingly capable of plaguing almost anyone in all cultures and ethnic groups. It is a very common reason for physician visits; second only to the common cold. Back pain is also the second most recorded cause for absenteeism, with close to 120 million working days being lost each year due to back pain [1].

In most cases, the only visual aid to assist medical staff with back pain assessment are 2-D "pain drawings" (Fig. 1), on which the patient is asked to mark the distribution and type (this being either symbol [2], [3] or color-coded [4]) of the pain being suffered. Studies have indicated that patient drawings have high completion rates, and in conjunction with a psychological evaluation, allow the physician to assess whether the pain is anatomic or non-anatomic [1]-[3].

The focus of the work described in this paper has been the augmentation of the traditional 2-D pain drawing with a novel, computer-based, 3-D version-in the anticipation that electronic 3-D pain drawings will increase the ease with which patients record their own pain, as well as provide a tool for pain data collection and monitoring of back pain clinicians.

\section{3-D PAIN DRAWINGS}

After consultations with clinicians and patients alike, it was felt that a patient would be able to better map the pain that they were experiencing onto a 3-D model of themselves rather than a 2-D one. Using a front and rear 2-D projection of a human torso makes it difficult to map pain that is occurring at the edges of the representation. A 3-D model provides a continuous body surface which allows a practitioner, or indeed a patient, to accurately record pain. Moreover, by being able to interact with the environment, anomalies caused by 2-D depth perception are removed and thus, consistency is increased.

In terms of back pain, an experimental study using 3-D for reconstructing spinal cord injuries reinforced the power of 3-D in medical practice. 3-D virtual images were constructed from performing computerized medical scans [5]. In this case, 3-D images were extremely beneficial because the models could be observed from many different viewpoints. Rotation and zooming features were combined to allow observer navigation within the tissue. 3-D navigation provided depth perception, so spatial relationships of features could be better revealed. The same feature benefit was anticipated from devising a 3-D adaptation of pain drawings.

Manuscript received July 27, 2006.

G. Ghinea, S. Kent, and G. S. Chana are with the School of Information Systems, Computing and Mathematics, Brunel University, Uxbridge UB8 3PH, U.K. (e-mail: george.ghinea@brunel.ac.uk; Simon.Kent@brunel.ac.uk; gurudain.chana@brunel.ac.uk).

A. O. Frank is with Northwick Park Hospital, Harrow HA1 3UJ, U.K. (e-mail: andrew.frank1@btinternet.com).

Digital Object Identifier 10.1109/TITB.2006.889710 


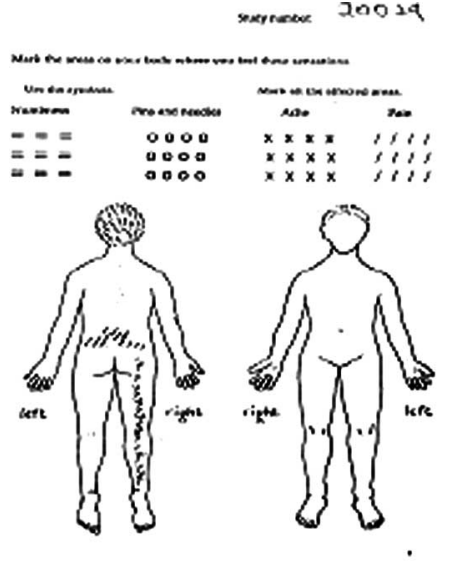

Fig. 1. Completed 2-D pain drawing.

\section{PROTOTYPE DESIGN}

The design of the prototype was conducted in collaboration with a team of clinicians from the rheumatology department of Northwick Park Hospital, London-potential users of the system. In brief, the identified requirements were to:

- provide a 3-D model of the human body;

- provide fully navigational controls enabling the ability to zoom, rotate, drag for depth perception;

- allow individually selectable regions of the body;

- use color to represent different types of pain;

- allow the details to be saved for later analysis by physician and for record keeping on a patient's file;

- avoid the use of specialized hardware or software.

It was thus felt that it would be useful if the prototype application could be delivered using a web-based approach. Macromedia Director was used to produce the 3-D Pain Visualization application that was distributable via the web.

To date, the interface has been used on a Windows XP PC, an Apple machine running OS X and a Linux PC. In our prototypical implementation, the application server comprised an Apache server configured to execute PHP (hypertext preprocessor) and a pain database implemented using MySQL, based on previous work of ours [6].

The 3-D model was developed using 3-D Studio Max 7. Based on earlier work [2], [3], we color-coded five different types of pain (burning, aching, stabbing, pins and needles, and numbness). Initially, a model was developed of an upright human body, but later on, a sitting version has also been created. Although a standing body might seem more natural, work has previously been undertaken to use a PDA to allow wheelchair users to record the pain that they experience during everyday wheelchair use [6].

This sitting model was more appropriate for this type of user. For the purpose of this paper, the use of different models demonstrates the flexibility of the system to allow alternative 3-D models to be used according to the needs of the patient. This is to make the patient's task as simple as possible by providing a realistic copy of their torso. An example of a 3-D model with the developed interface is shown in Fig. 2.

\section{EVALUATION}

\section{A. Clinical Evaluation}

Two clinicians, who used existing 2-D pain drawings, were asked to review the prototype: a back-pain specialist and a physiotherapist. Both

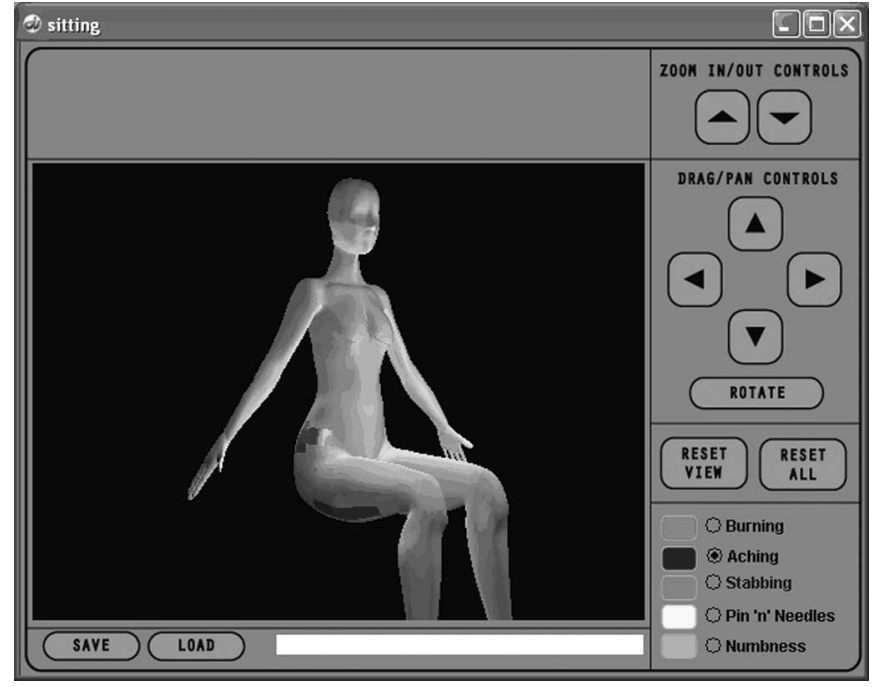

Fig. 2. Seated avatar for collection of pain data from a wheelchair user.

approved of the approach and agreed that the prototype would be useable in a clinical environment. Their observations can be summarized as follows.

1) Positive observations

a) 3-D interface covered almost all aspects of existing pain drawings.

b) The levels of detail and navigation control were impressive.

2) Negative observations

a) 3-D models needed enhancing to represent some pain locations. For example, some patients experienced pain on the outside of the hand, and there was no corresponding region on the models developed.

b) Only one type of pain could be recorded per region.

c) Colors used could be an issue for visually impaired or color blind patients.

\section{B. Patient Evaluation}

Thirty patients were asked to use the 3-D prototype for $10 \mathrm{~min}$ and to complete a questionnaire in order to evaluate the usability of the system. In general, the results were positive. Users were very satisfied with the navigation (68\% of users) and layout (87\%) of the interface. The context sensitive help (tooltips) that were provided, as users hovered over areas of the interface, was received with some ambivalence. Most participants $(68 \%)$ were satisfied with the color scheme, although this was the only question for which negative feedback was received, correlating with the view of the clinicians. A strong preference was expressed by all users for "rollover feature" in which pain areas were highlighted in grey as the mouse pointer was moved over the avatar to help the user to correctly record the pain in the correct place. Lastly, users were asked as to what would they like to improve regarding the system. The majority (57\%) stated that the regions should have been smaller, so as to pinpoint the pain they were experiencing more accurately.

\section{CONCLUSION}

This paper has described a 3-D pain drawing solution to address the limitation of existing 2-D approaches. Evaluation has shown that both general users and medically qualified practitioners perceive the 
3-D approach as positive, and it was found easy to use by both groups. However, the need for further development has also been identified.

Improvement is clearly needed in respect of allowing multiple types of pain to be recorded for a particular region of the body and using configurable color maps. Future work will also entail the porting of the prototype to wireless platforms, as well as the augmentation with animated 3-D versions potentially capable of displaying pain variation across time. All these efforts form an integral part of our thrust to build enhanced clinical wireless solutions.

\section{REFERENCES}

[1] A. O. Frank and L. H. De Souza, "Conservative management of low back pain," Int. J. Clin. Practice, vol. 55, no. 1, pp. 21-23, 2001.

[2] H. Parker, P. L. Wood, and C. J. Main, "The use of the pain drawing as a screening measure to predict psychological distress in chronic low back pain," Spine, vol. 20, no. 2, pp. 236-243, 1995.

[3] K. M. Prkachin, I. Schultz, J. Berkowitz, E. Hughes, and D. Hunt, "Assessing pain behaviour of low-back pain patients in real time: Concurrent validity and examiner sensitivity," Behav. Res. Therap., vol. 40, no. 5, pp. 595-607, 2002.

[4] R. Masferrer, V. Prendergast, and P. Hagell, "Colored pain drawings: Preliminary observations in neurosurgical practice," Eur. J. Pain, vol. 7, no. 3, pp. 213-217, 2002.

[5] B. S. Duerstock, C. L. Bajaj, V. Pascucci, D. Schikore, K. N. Lin, and R. B. Borgens, "Advances in three-dimensional reconstruction of the experimental spinal cord injury," Comput. Med.Imag. Graph., vol. 24, no. 6, pp. 389-406, 2000.

[6] T. Serif and G. Ghinea, "Recording of time-varying back-pain data: A wireless solution," IEEE Trans. Inf. Technol. Biomed., vol. 9, no. 3, pp. 447-458, Sep. 2005.

\section{Collaborative Work During Interventional Radiological Procedures Based on a Multicast Satellite-Terrestrial Network}

Lefteris G. Gortzis, Homer Papadopoulos, Theo A. Roelofs, Stefan Rakowsky, Dimitris Karnabatidis, Dimitris Siablis, Constantinos Makropoulos, George Nikiforidis, and Georgi Graschew

\footnotetext{
Abstract-Collaboration is a key requirement in several contemporary interventional radiology procedures (IRPs). This work proposes a multicast hybrid satellite system capable of supporting advanced IRP collaboration, and evaluates its feasibility and applicability. Following a detailed IRP requirements study, we have developed a system which supports IRP collaboration through the employment of a hybrid satellite-terrestrial network, a prototype multicast version of wavelet based interactive communication system (WinVicos) application, and a partition aggregation and conditional coding (PACC) wavelet codec. A semistructured questionnaire was also used to receive evaluative feedback from collaborating participants. The departments of interventional radiology of University Hospital of Patras, Greece and of Charite Hospital of Berlin, Germany have been connected on the system. Eight interventional radiologists and a vascular surgeon participated periodically in three satellite-terrestrial "fully collaborative" IRPs (average time $90 \mathrm{~min}$ ) of high complexity and in four terrestrial educational sessions with great success, evidenced by considerable improving the IRP outcomes (clinical and educational). In case of high complexity, where the simultaneous presence of remote interventional expert and/or surgeon is required, advanced collaboration among staff of geographically dispersed
}

international centers is feasible via integration of existing networking and other technologies.

Index Terms-e-health, multicast collaboration, radiology, satellite, wavelet encoding.

\section{INTRODUCTION}

Modern interventional radiological procedures (IRPs) require ad hoc multidisciplinary collaboration to improve the clinical and educational outcomes [1].

The real-time IRP collaboration demands effective visual reconstruction of remote Catheterization Lab (CL), data utilization features, satisfactory bandwidth, a clinically accepted video compression, and an integrated scenario. However, the few existing collaborative systems are not capable of supporting the main requirements, as many challenges limit their effectiveness [2], [3], i.e., hybrid networking [4], poor bandwidth [5], end-to-end delay [6], [7], and imaging quality [8].

This work aims to support collaboration during real-time IRPs among experts located at international referral centers worldwide by utilizing a satellite-terrestrial system. This system addresses the previous challenges by multicasting the required wavelet-based video feeds and the use of interactive features within a common multiscreen interface. The system's feasibility and applicability has been evaluated in three satellite-terrestrial "fully collaborative" IRPs with an average duration time of $90 \mathrm{~min}$ and in four terrestrial collaborative educational sessions.

\section{MATERIALS AND METHODS}

\section{A. System Overview}

The present system comprises four collaborative nodes, which are located at three terrestrial sites: two nodes at CL of the University Hospital of Patras, Patras, Greece, one node at Charite Hospital, Berlin, Germany, and one intermediate node at National Centre for Scientific Research (NCSR) Demokritos, Athens, Greece, as shown in Fig. 1.

To support IRP collaboration, the system employs a common multiscreen interface, and multicasts simultaneously the appropriate video feeds. These video feeds are presented in pop-up windows, as shown in Fig. 2. Particularly, the video feeds are able to display 1) the angio-unit output signal, which is captured to support the visual reconstruction of the intervention area; 2) the CL ceiling camera output signal, which is captured to support the CL environment visual reconstruction, e.g., hand movements of the interventionist performing the procedure etc.; 3 ) the document camera output signal, which is captured to visualize the MRI, or MRCP; and 4) the collaborators' cameras output signals, which are captured separately to support conference capabilities.

It should be noted that the video transmission parameters can be configured separately according to each node's capabilities (ten different frame parameters ranging from $128 \times 96$ to $640 \times 480$ are supported).

Manuscript received September 1, 2006; revised December 11, 2006.

L. G. Gortzis and G. Nikiforidis are with the Telemedicine Unit, Department of Medical Physics, University of Patras Medical School, 26500 Patras, Greece (e-mail: gortzis@med.upatras.gr).

H. Papadopoulos and C. Makropoulos are with the Division of Applied Technologies, National Centre for Scientific Research "Demokritos," 15310 Athens, Greece.

T. A. Roelofs, S. Rakowsky, and G. Graschew are with the Surgical Research Unit OP 2000, Robert-Roessle-Klinik and Max-Delbrueck Center for Molecular Medicine, University Hospital Charité, Humboldt University of Berlin, 10117 Berlin, Germany.

D. Karnabatidis and D. Siablis are with the Department of Interventional Radiology, University Hospital of Patras, Patras 26500, Greece.

Digital Object Identifier 10.1109/TITB.2007.894724 